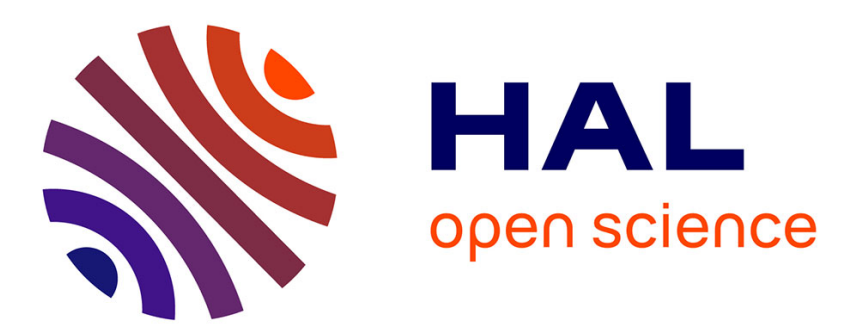

\title{
Jérôme, Augustin, Boèce. Prolégomènes à toute traduction médiévale qui voudra se présenter comme science \\ Anna Svenbro
}

\section{- To cite this version:}

Anna Svenbro. Jérôme, Augustin, Boèce. Prolégomènes à toute traduction médiévale qui voudra se présenter comme science. Cahiers d'Etudes Hispaniques Médiévales, 2019, Penser la traduction au Moyen Âge, 41, pp.23-36. 10.3917/cehm.041.0023 . hal-02441444

\section{HAL Id: hal-02441444 \\ https://hal.science/hal-02441444}

Submitted on 25 Mar 2020

HAL is a multi-disciplinary open access archive for the deposit and dissemination of scientific research documents, whether they are published or not. The documents may come from teaching and research institutions in France or abroad, or from public or private research centers.
L'archive ouverte pluridisciplinaire HAL, est destinée au dépôt et à la diffusion de documents scientifiques de niveau recherche, publiés ou non, émanant des établissements d'enseignement et de recherche français ou étrangers, des laboratoires publics ou privés. 


\section{Jérôme, Augustin, Boèce}

\section{Prolégomènes à toute traduction médiévale qui voudra se présenter comme science}

Anna Svenbro

\section{Introduction}

Dans le tableau Saint Jérôme dans son étude d'Antonello de Messine, l'auteur de la Vulgate contemple le livre entre ses mains, et fait face à celui-ci comme la Vierge Marie à l'Archange Gabriel dans une Annonciation ${ }^{1}$. Le studiolo, les livres sur les étagères, comme celui entre les mains du « Saint Docteur », se présentent comme autant de cadres de déchiffrement du monde, de fenêtres renvoyant, par jeu de miroirs, au monde extérieur à l'arrière-plan comme à celui du spectateur du tableau ${ }^{2}$ : beau rappel pictural de la nature profondément théorique de l'œuvre du patron des traducteurs !

La postérité a souvent retenu du rapport à l'Antiquité des traducteurs et penseurs médiévaux ayant traité de traduction - du moins jusqu'à Thomas d'Aquin - le fait que ceux-ci s'appuyaient sur les adages antiques de Cicéron et d'Horace via Jérôme comme sur un ensemble parcellaire et hétérogène érigé «au doigt mouillé » en corpus de règles générales; un tel constat est à nuancer ${ }^{3}$. Certes, ce rapport est infusé par des textes de Cicéron et Horace, bien sûr, mais également d'AuluGelle, Pline le Jeune, Quintilien, etc., où la traduction est à l'état de proto-théorie. Mais il est surtout tributaire de lectures qu'ont pu en faire non seulement Jérôme, mais encore Augustin et Boèce. Ce substrat riche, complexe, dense est bien loin d'une simple série d'adages. Il impose sa marque aux traducteurs médiévaux, quitte à devenir pour eux une « seconde nature » plus ou moins consciente, et à revêtir un statut "paradigmatique », avec un ensemble de problèmes-types et de solutions, si l'on reprend la terminologie forgée par Kuhn dans La Structure des révolutions scientifiques ${ }^{4}$.

Sur le tableau d'Antonello, comme dans toute la tradition picturale, Jérôme est représenté avec les attributs cardinalices. Or, s'il a été un temps conseiller du pape Damase, Jérôme n'a jamais été cardinal, pas plus que ne l'ont été son contemporain Augustin (évêque d'Hippone), ni Boèce un siècle après eux ! Pourtant, ces trois figures sont cardinales à plus d'un titre : figures principales (en latin cardinales) de l'histoire des premiers temps du christianisme et de l'histoire de la traduction, fondatrices d'une nouvelle conception de l'acte de traduire, elles sont aussi des charnières (cardines) entre deux époques et deux systèmes discontinus en matière de théorisation. Dans leur sillage, on met à distance les prémisses « cicéroniennes » pour construire un système « chrétien », si l'on reprend l'alternative posée par Jérôme dans sa Lettre 22 à Julia Eustochium ${ }^{5}$. On présentera ainsi à travers ces figures les premières grandes tentatives de théoriser la traduction à la charnière de l'Antiquité et du Moyen-Âge, les principaux débats auxquels elles ont pris part et la manière dont elles ont posé les fondements de la traduction médiévale. Car avec Jérôme, Augustin puis Boèce, la traduction devient l'objet d'une « science », au sens latin de scientia, c'est à dire un savoir raisonné, normatif et validé par une institution.

\footnotetext{
${ }^{1}$ Penny Howell Jolly, "Antonello da Messina's "Saint Jerome in His Study" An Iconographic Analysis", The Art Bulletin, vol. 65 (2), juin 1983, vid. p. 238-253.

${ }^{2}$ Georges Perec, Espèce d'espaces, Paris : Galilée, 1974, vid. p. 118.

${ }^{3}$ Louis G. KeLly, "Medieval Philosophers and translation", in Hans-Josef NIEDEREHE \& Ernst Frideryk Konrad Kowrner (éd.), Studies in the History of the Language Science, Amsterdam : John Benjamins Publishing, 1990, vid. p. 205-218.

${ }^{4}$ Thomas S. KuHN, La structure des révolutions scientifiques, Paris : Flammarion, vid. 1983, p. 11.

${ }^{5}$ Saint Jerome, Correspondance, trad. Jérôme LabourT, Paris : Les Belles Lettres, 2002, tome I, 22, vid. p.145.
} 


\title{
La « science » de la traduction chez Jérôme, Augustin et Boèce : avant la triade observation-lois-expérimentation, la triade décrire-prescrire- prospecter
}

\section{Les ambiguïtés de la description}

Si l'on s'intéresse à la dimension descriptive de l'activité théorique chez Jérôme, Augustin et Boèce, on s'aperçoit que celle-ci est certes parcellaire, mais loin d'être introuvable. Elle s'insère dans un système plus large, celui des procédés rhétoriques. Viri eloquentissimi et doctissimi, Jérôme, Augustin et plus tard Boèce sont les purs produits de l'éducation libérale du Bas-Empire, centrée sur les cultures latine et grecque, avec, au sommet, la rhétorique, accomplissement de tous les savoirs.

Jérôme sous-titre la Lettre 57 à Pammaque De optimo genere interpretandi, « sur la meilleure façon de traduire »: il place directement sa réflexion sous les auspices de la rhétorique, paraphrasant le titre cicéronien De optimo genere oratorum, « Du meilleur genre d'orateurs ». La référence n'est pas innocente : à l'époque, quand on parle de traduction, il y a toujours inuentio, détermination des mots et du style appropriés pour la rendre acceptable selon le contexte dans lequel elle est produite. Boèce, lorsqu'il décrit une partie de son projet de traduction du corpus aristotélicien dans la préface à son commentaire de la doctrine aristotélicienne du jugement, s'accorde toujours au contexte et aux canons de la rhétorique latine :

\begin{abstract}
Je veux traduire en latin toute l'œuvre d'Aristote, du moins ce que je pourrai en atteindre et présenter consciencieusement en langue latine toutes ses déclarations. [...] De plus, je voudrais aussi traduire et expliquer tous les dialogues de Platon et les présenter accommodés au latin ${ }^{6}$.
\end{abstract}

Augustin intègre quant à lui ses remarques sur la traduction à une réflexion sur la rhétorique en contexte chrétien, dans les deuxième et troisième livres de De Doctrina Christiana, ouvrage visant à guider les fidèles désireux d'analyser les textes bibliques, de manière à y trouver (inuenire) de quoi susciter leur foi. Il y fait un plaidoyer pour la réflexion sur le, ou plutôt, sur les textes bibliques, c'est-à-dire les originaux hébraïques et grecs par rapport à leurs traductions latines. Le lecteur des Saintes Écritures réfléchit à leur(s) traduction(s) en s'appuyant sur sa culture linguistique, grammaticale et rhétorique.

\footnotetext{
Sa science des langues lui évite d'être arrêté par les mots et les expressions inconnues, sa connaissance d'un certain nombre de réalités indispensables lui évite d'ignorer le sens et la valeur de celles auxquelles il est fait appel pour des comparaisons, avec l'aide de l'exactitude de manuscrits corrigés avec soin et compétence: qu'ainsi armé il s'attaque à la discussion et à l'explication des passages ambigus des Écritures ${ }^{7}$.
}

Les Écritures sont ici le témoignage de la Parole divine, à la fois unique, car venant d'un Dieu unique, et divers, empruntant les récits des prophètes sur le lien entre ce Dieu unique et son Peuple, puis exposant, dans le cadre d'une multiplicité contextualisée, les témoignages de la mort et de la résurrection du Christ: «Dieu a parlé une fois, deux fois j'ai entendu ${ }^{8}$ ». Augustin donne à partir d'elles une première définition de la traduction :

De là vient que la divine Écriture, qui porte si souvent secours au infirmités de la volonté humaine, à partir d'une langue unique qui lui permit opportunément de se répandre sur toute la terre, se fit connaître aux nations pour leur salut, au loin et largement, par les diverses langues des interprètes : en la lisant, ces nations ne cherchent rien d'autre que les pensées et la volonté de ses auteurs et, par elles, la volonté

\footnotetext{
${ }^{6}$ Boece, cité par Hans Von CAMPEnhausen, Les Pères Latins, traduit de l'allemand par C.A. MoreaU, Paris : Éditions de l'Orante, 1969, vid. p. 331-332.

${ }^{7}$ SAINT Augustin, De Doctrina Christiana, III, 1, in Oeuvres, trad. Jean-Louis DuMAS, Sophie DuPUY-TRUDELLE, Jean-Yves Boriaud et Patrice CAMBronne, sous la direction de Lucien JERPHAGNON, Paris : Gallimard, La Pléiade, 1998, tome III, vid. p. 74.

${ }^{8}$ Ps 62:11.
} 
de Dieu, conformément à laquelle ont parlé, croyons-nous, de tels hommes9 .

Pour Augustin, les Écritures se sont fait connaître par la diversité des signes et des idiomes employés par les interprètes, et l'ont ainsi transcendée.

Pourtant, ces théorisations manquent d'ordre méthodologique, chez Augustin, comme chez Jérôme et Boèce. Il n'y a pas vraiment de description des procédés de traduction. On ne peut constater que la rareté des tentatives de décrire les méthodes de transfert interlinguistique des éléments signifiants d'un texte original vers un texte d'arrivée. La méthode est rivée à la rhétorique antique, comme le montre cette phrase de Jérôme dans sa Lettre à Pammaque :

Pour s'asservir au cas et aux figures, le style, qui pouvait manifester telle idée en un bref langage, malgré de longs détours ou périphrases, ne parvient qu'à peine à l'exposer ${ }^{10}$.

Lorsque Jérôme pointe ce que nous appellerions de nos jours une absence de recatégorisation pour coller aux catégories grammaticales du texte de départ, le phénomène n'est pas analysé pour luimême, mais dans le cadre de l'inuentio cicéronienne : il s'agit toujours de déterminer les mots et le style appropriés pour rendre la traduction acceptable selon les cadres rhétoriques latins.

\section{Fortune et limite des prescriptions}

Plus visibles sont en revanche les prescriptions qui envisagent de manière normative la tâche du traducteur. Ce sont surtout Jérôme et Boèce qui se font théoriciens de la traduction par l'exemple, à partir de leur propre pratique. Augustin théorise lui dans le cadre d'une herméneutique plus générale, prenant l'occasion de professer ce qu'il faut faire et ne pas faire.

Les premières prescriptions tournent autour de l'expertise du traducteur, sa science et sa légitimité : le traducteur les tire de son savoir théorique et technique. Ainsi, ce sont les vastes compétences linguistiques, exégétiques et théologiques du uir trilinguis qu'est Jérôme qui attirent l'attention du pape Damase et amènent ce dernier à lui confier l'établissement de la Versio vulgata, traduction latine autorisée de la Bible censée remplacer les traductions existantes.

Mais si le traducteur est certes défini par sa science, il est d'abord défini en creux, au travers de polémiques par lesquelles les parties prenantes érigent leurs adversaires en repoussoirs : on a avant tout des tentatives de définir ce qu'est un mauvais traducteur ! La Lettre 57 à Pammaque dresse une galerie de portraits peu amènes des adversaires de Jérôme (Rufin et Aquila) : ils ont « le mauvais goût des illettrés », leurs traductions «sonnent mal en latin». Entre deux invectives, le bon traducteur est ainsi distingué :

Je n'entends pas blâmer chez un chrétien ordinaire l'impéritie du langage (plût à Dieu que nous fassions nôtre l'adage socratique: «je sais que je ne sais rien», et celui d'un autre sage: «connais-toi toimême !»), car j’ai toujours vénéré, non pas la rusticité verbeuse, mais la sainte simplicité ${ }^{11}$.

Même si le ton employé est moins acerbe, on peut retrouver les mêmes jugements chez Augustin :

Les hommes, cependant, sont d'autant plus choqués qu'ils ont moins de capacités, et ils ont d'autant moins de capacités qu'ils veulent paraître plus savants ${ }^{12}$.

Pourquoi de telles exigences ? Jérôme comme Augustin s'inscrivent «dans la tradition des litterati, rattachés, comme Macrobe, par toutes leurs fibres aux goûts de l'école antique ${ }^{13}$. » Mais à ce legs

\footnotetext{
${ }^{9}$ SAint Augustin, ibid. II, 5, p. 35. C'est nous qui soulignons.

${ }^{10}$ SAINT Jerome, Correspondance, trad. Jérôme LABOURT, Paris : Les Belles Lettres, 2002, vid. tome III, 57 , p. 61.

${ }^{11}$ SAINT JÉRÔME, ibid. p. 71.

${ }^{12}$ SAINT Augustin, ibid. II, XII, 20, p. 45.

${ }^{13}$ Michel BANNIARD, «Jérôme et l'elegantia d'après De optimo genere interpretandi », in Yves-Marie DuvAL (éd.), Jérôme, entre l'Occident et l'Orient (Actes du colloque de Chantilly, septembre 1986), Paris : Études augustiniennes, 1989 , vid. p. 322.
} 
païen s'ajoute celui des Septante. Les fondements de la légitimité du traducteur sont ici les suivants : le fait que tous les traducteurs impliqués dans l'élaboration de la version des Septante aboutissent à la même traduction implique qu'ils connaissent leur affaire. Leur expertise est validée par une instance extérieure (ici Eléazar, le Grand Prêtre de Jérusalem). Le public de la traduction légitime les traducteurs du fait de leur expertise, et valide la traduction comme étant le fruit du travail d'experts. Or, Augustin note :

S'ils ont cependant comparé leurs traductions de manière que leurs travaux et leurs jugements ne résulte qu'une voix, même ainsi, il n'est pas un homme, quel que soit son talent, qui puisse ou qui doive aspirer à corriger de son côté ce sur quoi se sont accordés tant de savants ${ }^{14}$.

L'expertise des traducteurs s'inscrit dans un processus délibératif, l'objectivité due au décentrement du jugement de chaque traducteur au sein d'une décision collective étant préférable à la subjectivité (peut-être partielle, partisane) d'un seul, dont l'expertise ne vaut que si elle est validée par celle d'autrui et celle de l'institution: d'où les réserves d'Augustin par rapport à la Vulgate, fruit du travail solitaire de Jérôme.

«Orator antequam dictor ${ }^{15}$ », «homme de prière avant d'être homme de discours » (et on appréciera le jeu sur la polysémie d'orator...): pour Augustin, comme l'orateur chrétien, le traducteur doit combiner ses compétences linguistiques avec les qualités pratiques et morales chrétiennes. D'aucuns ont pu voir, comme le fait Douglas Robinson dans son article consacré aux fondations ascétiques de la traduction occidentale ${ }^{16}$, la liste des qualités requises pour légitimer la tâche du traducteur comme autant de chapitres d'une règle monastique : crainte, piété, connaissance, force d'âme, miséricorde, pureté et enfin sagesse...

\section{La traduction à l'heure des innovations prospectives}

Nous pouvons enfin voir chez Jérôme et Augustin une démarche prospective, critique, qui ne s'arrête pas à ce qui est traduit, ce qu'il faut traduire et comment il faut traduire. Ils ne se contentent pas de constater la différence des traductions entre elles; celles-ci font sens et sont sources d'interprétations fécondes.

Jérôme, dans sa Lettre 57 à Pammaque, ne cesse de pointer les différences entre la version hébraïque de l'Ancien Testament, la traduction grecque des Septante et les citations qui en sont faites dans le Nouveau Testament. Est-ce pour s'autoriser à prendre des libertés dans ses propres traductions ? La réalité est bien plus complexe.

Quelle distance n'y a-t-il pas entre le texte de l'évangéliste et la traduction des Septante ! C'est trop clair. Mais, dans l'original hébreu, si le sens est le même, les mots sont disposés à l'envers et presque différents. [...] Qu'ils accusent l'Apôtre de falsification, parce qu'il n'est d'accord ni avec l'hébreu ni avec les LXX traducteurs et - ce qui est plus grave - parce qu'il fait erreur sur le nom (en effet, au lieu de Zacharie, c'est Jérémie qu'il a cité) ; mais que l'on se garde bien de traiter ainsi le disciple du Christ, qui s'est soucié non pas de donner la chasse aux mots et aux syllabes, mais d'exprimer des maximes doctrinales ${ }^{17}$.

En raison du statut scripturaire des textes, la critique s'interdit d'être négative. Or, les écarts, les tensions des textes les uns par rapport aux autres sont revendiqués. Jérôme ne critique pas au sens négatif les inexactitudes de la version des Septante ou des citations des Évangélistes. Au contraire, les déviations sont assumées, louées comme créatrices et autant d'occasions de déployer les infinies

\footnotetext{
${ }^{14}$ Saint Augustin, De Doctrina Christiana, II, XII, 20, trad. Madeleine Moreau, Bibliothèque augustinienne 11/2, Paris, 1998.

${ }^{15}$ Saint Augustin, ibid. IV, XV, 32, in Oeuvres, trad. Jean-Louis Dumas, Sophie DuPuY-Trudelle, Jean-Yves Boriaud et Patrice CAMBronNe, sous la direction de Lucien JERPHAGnON, Paris : Gallimard, La Pléiade, 1998, tome III, vid. p. 129.

${ }^{16}$ Douglas RoBInson, "The Ascetic Foundations of Western Translatology: Jerome and Augustine",Translation in Literature, vol. 1, Edinburg: Edinburg University Press, 1992, vid. spec. p. 18.

${ }^{17}$ SAINT JÉRÔME, ibid. p. 63.
} 
nuances de la Parole.

Pour Augustin, dans De Doctrina Christiana tout comme le livre XVIII de De Civitate Dei, la diversité des langues et des traductions est un postulat: pour lui, la confusion des langues est dépassée par la traduction, la diversité par l'unité, unité qui n'est pas synonyme d'unicité. La pluralité des traductions, loin d'être source de confusion, est source de clarté.

L'examen de plusieurs versions a souvent éclairé, en effet, les expressions obscures [...] Sont également d'un très grand secours l'examen et la discussion de plusieurs traductions, à partir de la confrontation de diverses éditions ${ }^{18}$.

Pourquoi les différences entre les traductions font-elles sens pour Augustin ? Pourquoi les originalités ne mènent-elles que mieux à l'origine ? Dans De Civitate Dei, à propos de l'élaboration de la version des Septante, il jette les fondements décisifs d'un travail de critique des traductions :

Or, assurément, il a pu, de son autorité divine, exprimer un autre sens, comme si le prophète avait annoncé l'un et l'autre sens. Il a pu encore dire la même chose en d'autres termes, de sorte qu'à défaut des mêmes mots le sens apparaisse bien le même aux esprits droits. Il a pu enfin supprimer ou ajouter, afin de montrer par-là que dans ce travail il n'y avait point servitude de l'homme, soumettant le traducteur aux mots, mais plutôt puissance divine remplissant et guidant l'intelligence du traducteur ${ }^{19}$.

\section{Que traduire ? La traduction entre l'esprit et la lettre}

\section{Du genre au sens et de l'esprit à la lettre : la mise à distance des pratiques cicéroniennes}

Concernant l'opération de traduction en elle-même, Jérôme n'est pas la caisse de résonance des adages cicéroniens que beaucoup ont voulu faire de lui. Sa réflexion sur la traduction est au contraire une mise à distance de ces adages par glissements successifs, que Boèce poussera encore plus loin.

Pour faire bref ${ }^{20}$, le traité de Cicéron s'intitule De optimo genere oratorum, «Du meilleur genre d'orateurs ». Celui de Jérôme, De optimo genere interpretandi, «Du meilleur genre de traduction». Ce n'est pas en orateur que Jérôme traduit, mais en interprète : tout le contraire de Cicéron. D'une part Cicéron écrit :

J'ai en effet traduit des deux plus éloquents des Attiques, Eschine et Démosthène, les deux discours les plus célèbres et qui se répondent; et je les ai traduits non en interprète, mais en orateur (nec converti ut interpres, sed ut orator), avec la même présentation des idées et des figures, en adaptant les mots à notre propre langue. Pour ceux-ci je n'ai pas jugé nécessaire de les rendre mot par mot (non uerbum pro uerbo necesse habui reddere), mais j'ai conservé dans son entier le genre des expressions et leur valeur (sed genus omne uerborum uimque seruaui). Je n'ai pas cru en effet que je dusse en rendre au lecteur le nombre, mais en quelque sorte le poids ${ }^{21}$.

C'est une fidélité aux éléments de la pragmatique du texte source qui compte pour Cicéron. Jérôme, lui, soutient :

Ce n'est pas un mot par un mot, mais une idée par une idée que j'exprime (non uerbum e uerbo sed

\footnotetext{
${ }^{18}$ SAInt Augustin, ibid. II, 12, p. 42-46.

${ }^{19}$ Saint Augustin, De Civitate Dei, XVIII, 43, in Oeuvres, trad. Jean-Louis Dumas, Sophie DuPUY-Trudelle, JeanYves Boriaud et Patrice CAMBronne, sous la direction de Lucien JERPHAGNON, Paris : Gallimard, La Pléiade, 1998, vid. tome II, p. 821-822.

${ }^{20}$ Pour plus de détails, voir Anna SVENBRO, «Théoriser la traduction à la fin de l'Antiquité et au début du Moyen-Âge : quelques glissements sémantiques », in Bernard BORTOLUSSI, Sophie MINON, Lyliane SZNAJDER, Traduire, Transposer, Transmettre dans l'Antiquité gréco-romaine, Paris : Picard, 2009, vid. p. 9-16.

${ }^{21}$ CiCEROn, Du meilleur genre d'orateur, V, 14-15 trad. Albert Yon, Paris : Les Belles Lettres, 1964, p. 111.
} 
sensum exprimere de sensu) $)^{22}$.

\title{
Fortune du fidus interpres : du traducteur servile au traducteur fidèle
}

On observe une mise à distance similaire chez Jérôme de l'adage que l'on trouve chez Horace, dans son Ars poetica :

Vous ferez d'une matière prise au domaine public votre propriété privée si vous ne vous attardez pas à faire le tour de la piste banale et ouverte à tous, si vous ne vous appliquez pas à rendre, traducteur trop fidèle / servile, le mot par le mot (nec uerbo uerbum curabis reddere fidus interpres), si vous ne vous jetez pas, en imitant, dans un cadre étroit d'où la timidité ou bien l'économie de l'œuvre vous interdiront de sortir ${ }^{23}$.

Ainsi, Jérôme écrit :

Horace, lui aussi, cet homme si fin et si docte, trace, dans son Art poétique, les mêmes règles à un traducteur lettré : 'tu ne te soucieras pas de rendre chaque mot par un mot, tout en restant fidèle interprète (nec uerbo uerbum curabis reddere fidus interpres) ${ }^{24}{ }^{2 . .}$

Chez Horace, on avait affaire à un traducteur servile, vilipendé parce qu'il n'apportait rien par rapport à l'original et n'était pas poète. Chez Jérôme, le traducteur fidèle est quant à lui loué pour son respect de l'esprit de l'original.

Boèce, quant à lui, va encore plus loin, dans sa préface à la seconde édition du commentaire de sa traduction de l'Isagogè de Porphyre :

\begin{abstract}
Je crains d'avoir commis la faute du 'fidèle interprète', quand j'ai rendu un mot, l'ai fait correspondre et l'ai exprimé par un autre (uereor ne subierim fidi interpretis culpam cum uerbum uerbo expressum comparatumque reddiderim). Voici la raison de cette entreprise : dans des textes où l'on cherche la connaissance des choses, ce n'est pas l'élégance du beau discours, mais la vérité incorrompue qu'il faut chercher. Pour cette raison, il me semble que j'aurais fait un grand progrès, si, dans les ouvrages de philosophie traduits en latin, grâce à l'honnêteté d'une traduction scrupuleuse, rien de plus ne fait défaut que ce qui fait défaut dans les textes des Grecs ${ }^{25}$.
\end{abstract}

La crainte formulée par Boèce est ironique, il assume sa servilité, et son projet de rendre un mot par un autre : la fidélité à la lettre, à la vérité de l'original, vient d'abord, et le bon usage latin passe au second plan.

\section{Naissance de l'objection préjudicielle en traduction}

Avec Boèce, un pas de plus est franchi dans la théorisation de la traduction : la sensibilité du signe est réhabilitée comme moyen de mettre au jour l'intelligible. La lettre ne tue plus : la chair vive du texte traduit est destinée à accueillir l'esprit de l'original grec voué à devenir lettre morte, du moins dans l'Occident latin. L'entreprise boétienne est humaine, mais tient d'une tentative de résurrection. Elle sera donc entachée de la suspicion de blasphème et/ou de mystification. La pratique boétienne est l'illustration éclatante des scrupules du traducteur face à une entreprise nécessaire mais impossible, œuvre de «faussaire» ou de «blasphémateur», pour reprendre l'alternative talmudique. L'objection préjudicielle chère à la traductologie moderne, mais qu'on retrouve au Moyen-Âge, chez un Roger Bacon entre autres, fait ici surface. Comme le souligne Jean-René Ladmiral :

Singulièrement, quand il s'agit de traduction, la réflexion commence d'abord par s'interroger sur la

\footnotetext{
${ }^{22}$ SAINT JEROME, ibid. p. 59.

${ }^{23}$ HorACE, Epîtres, trad. François VILlEneuve, Paris : Les Belles Lettres, 2002, p. 209.

${ }^{24}$ SAINT JEROME, loc. cit.

${ }^{25}$ BoECE, in Samuel BRANDT (éd.), Anicii Manlii Severini Boethii In Isagogen Porphyrii commentorum, editionis secundae, I, Corpus Scriptorum Ecclesiasticorum Latinorum - CSEL, vol. 48, Vienne et Leipzig : F. Tempsky, 1906, p. 135. C'est nous qui soulignons et traduisons.
} 
possibilité même de cette pratique qu'elle prend pour objet; bien plus, la tendance lourdement prédominante est de conclure à l'impossibilité théorique de traduire ! C'est là un paradoxe bien étrange et, semble-t-il, tout à fait propre à la traduction ${ }^{26}$.

Dans le cas de l'Antiquité tardive, Henri Meschonnic note que le sacré fait office de laboratoire de l'objection préjudicielle : il explore à travers les Écrits bibliques les limites, et donc les possibilités et les impossibilités de l'acte de traduire ${ }^{27}$.

Des siècles de vénération les ont rendus lourds de signification. Leur utilisation dans la liturgie inspire le respect et met un frein aux changements ${ }^{28}$.

Or, la dimension de changement impliquée par la traduction la place sous un jour qui est celui de la suspicion : pour certains, agent de la Révélation, elle est pour d'autres celui du blasphème. Dans le premier cas, on considère que la traduction est possible ; dans le deuxième, on préjuge de son impossibilité. L'oubli de l'original devient impossible : ainsi, celui de l'Hebraica ueritas pour Jérôme. Même si Augustin préconise de toujours traduire à l'aune de la version des Septante, lui aussi reconnaît cet héritage.

La Lettre 20 à Damase a ainsi pour sujet principal le mot hébreu Hosanna et son caractère difficilement traduisible. Jérôme choisit, non pas de passer outre et de nier l'altérité et l'intraduisibilité du terme, mais de l'affronter. L'exposé très détaillé de philologie hébraïque témoigne d'une recherche acharnée pour trouver la juste correspondance en latin avec le terme hébreu Hosanna. Finalement, cette recherche débouche sur un échec ; la difficulté de traduire est pourtant assumée, Jérôme ayant déjà remarqué dans une lettre précédente à Damase, la Lettre 18 :

Dans les Écritures, les mots ne sont pas simples, comme d'aucuns le pensent : très nombreux y sont les sens cachés. Autre est la signification de la lettre, autre celle du langage mystique ${ }^{29}$.

Lisons en contrepoint l'analyse d'Augustin à propos de l'intraduisibilité d'Hosanna, à laquelle s'ajoutent celles d'Amen, Alleluia et Racha.

Bien que nous trouvions souvent, dans les Livres, des mots hébreux non traduits, comme Amen, Alleluia, Racha, Osanna et quelques autres. Pour certains, comme Amen et Alleluia, c'est le caractère sacré de leur autorité qui leur a valu de conserver leur forme antique, bien qu'il ait été possible de les traduire ; d'autres comme les deux derniers, passent pour intraduisibles. Il est en effet des mots, dans certaines langues, qui ne peuvent entrer, par la traduction, dans l'usage d'une autre langue ${ }^{30}$.

L'ombre de l'objection préjudicielle plane aussi sur le projet boétien, même si Boèce, dans le domaine profane de la philosophie grecque, y apportera une solution à rebours de celle proposée par Jérôme et Augustin. Il y a contradiction flagrante dans ses objectifs présentés plus haut pour l'Isagogè de Porphyre et son projet décrit dans la préface à son commentaire de la doctrine aristotélicienne du jugement :

Tout ce qu'Aristote peut bien avoir écrit sur l'art difficile de la logique, sur le grave domaine des expériences morales et sur l'exacte intelligence des choses de la nature, voilà ce que je vais traduire en suivant un ordre logique. Et je veux rendre tout cela plus aisément compréhensible par mes éclaircissements. De plus, je voudrais aussi traduire et expliquer tous les dialogues de Platon et les

\footnotetext{
${ }^{26}$ Jean-René LADMIRAL, Traduire - Théorèmes pour la traduction, Paris : Gallimard, 1994, vid. p. 85.

${ }^{27}$ Henri MESCHONNIC, « Le calque dans la traduction », Cahiers internationaux de symbolisme, no 31-32, 1976, p. 6575 .

${ }^{28}$ Sherry SiMON, «Les traducteurs, propagateurs des religions », in Jean DELISLE, Judith WoODSWORTH (éds.), Les traducteurs dans l'histoire, Ottawa : UNESCO et Presses de l'Université d'Ottawa, 1995, vid. p. 163.

${ }^{29}$ SAINT Jerome, Correspondance, trad. Jérôme LABOURT, Paris : Les Belles Lettres, 2002, tome I, 18, vid. p. 66.

${ }^{30}$ SaInt Augustin, De Doctrina Christiana, II, XI, in Oeuvres, trad. Jean-Louis Dumas, Sophie DuPUY-TRUDELlE, Jean-Yves BoriAud et Patrice CAMBRONNE, sous la direction de Lucien JERPHAGNON, Paris : Gallimard, La Pléiade, 1998, vid. tome III, p. 42. C'est nous qui soulignons.
} 
présenter accommodés au latin $^{31}$.

Certes, la traduction chez Boèce a en partie pour vocation de se substituer aux textes grecs originaux en dispensant les latinophones de leur lecture ; devant l'impossibilité de la traduction à la lettre, un compromis est trouvé dans la pratique du commentaire. Or, cette solution adoptée par le «père du Moyen-Âge européen » est lourde de conséquences sur la traduction médiévale et son lien avec la pratique du commentaire.

\section{Le nouvel esprit traducteur : entre obéissance et menaces}

\section{Nouvelles hiérarchies et nouvelles partitions des textes à traduire}

Si les traducteurs sont soumis à un nouveau jeu de réflexions et de préceptes, leur tâche s'infléchit aussi dans leur rapport à l'autorité et aux institutions. C'est que les réflexions autour de la traduction revêtent un enjeu théologico-politique. En effet, de religion persécutée par les autorités impériales romaines, sans intérêts séculiers, le Christianisme, par le biais de l'Église, émerge comme nouvelle autorité et puissance temporelle prenant en charge un nouvel ordre séculier. La réflexion hiéronymienne a pour arrière-plan le besoin pour l'Église de maintenir le contrôle du dogme, et donc des traductions de la Bible. On rappellera les traces profondes que laissent sur Jérôme et Augustin le moment gnostique d'une part, et, d'autre part, les débats sur le manichéisme, le pélagianisme, l'origénisme, clos par l'autorité des conciles. À travers Jérôme, la traduction devient une «science »: si l'on s'en tient à l'acception latine du terme, un savoir raisonné, et surtout normatif. Cette «science » revêt une fonction politique de contrôle du transfert et de la réception des Écritures à travers leurs versions hébraïque et grecque vers les croyants de culture latine. Or, ce contrôle naissant ne concerne pas uniquement le Verbe dans le cadre d'un processus d'unification des textes bibliques. Il pèse aussi sur les traducteurs, la hiérarchie ecclésiastique s'assurant de leur conformité aux normes interprétatives en vigueur, et le traducteur apparaît comme potentiellement dangereux s'il diverge de ce que l'institution attend de lui. Jérôme, déchiré entre sa culture classique et sa mission chrétienne, est aussi tiraillé entre son appartenance à l'Église et sa vision de la traduction, très indépendante par rapport à l'institution ecclésiastique, qui coalise contre lui bon nombre d'oppositions qui contribuent à le mettre un temps à l'écart.

Dans la Lettre 57 à Pammaque, Jérôme répond à de multiples attaques et cabales; il y illustre le caractère non seulement polémique, mais encore défensif de sa théorisation. Jérôme écrit pour « répondre à la langue d'un sot », réfuter les griefs de son ennemi Rufin sur une traduction qu'il a faite et qui est réputée infidèle à l'original. En effet, Epiphane, évêque de Salamine, a auparavant adressé à l'évêque Jean de Jérusalem une lettre en grec au ton acerbe sur divers points dogmatiques controversés. Un moine dans l'entourage de Jérôme, non helléniste, prie Jérôme de la lui traduire en latin. Jérôme s'acquitte rapidement de la tâche, en marge d'un exemplaire de la lettre, à condition expresse que cette traduction reste confidentielle; dix-huit mois plus tard, en dépit de ces précautions, la traduction est rendue publique. Ses adversaires lui reprochent son infidélité au texte original et la manière cavalière de traduire les formules honorifiques. La réponse de Jérôme à la publicité de sa traduction contre son gré est cinglante :

Tant que je ne publie pas mes cogitations, même ce qui est mal dit n'est pas crime ; bien mieux : ce n'est même pas mal dit, puisque les oreilles du public l'ignorent ${ }^{32}$.

La sphère privée est envisagée en négatif : ce qui est mal dit, ou traduit, se rapproche du non-dit ( « ce n'est même pas mal dit») du fait de sa non-appartenance au domaine public. Il y a bipartition entre domaine public et sphère privée, bipartition totalement absente de la conception romaine de la traduction. Même si les traductions destinées au domaine public tournent toujours les textes vers

\footnotetext{
${ }^{31}$ Boece, cité par Hans Von CAMPENHAUSEn, loc. cit. C'est nous qui soulignons.

${ }^{32}$ SAINT Jerome, Correspondance, trad. Jérôme LABOURT, Paris : Les Belles Lettres, 2002, vid. tome III, 57 , p. 58.
} 
l'extérieur, c'est désormais à l'aune des normes interprétatives élaborées par l'institution. Ces traductions coexistent avec des traductions «pour le tiroir», marquées par la confidentialité et le secret, dont la divulgation peut comporter d'énormes risques pour le traducteur, Jérôme ayant connu la disgrâce en marge de cet épisode.

\section{Le traducteur, figure désormais ambiguë}

Les lettres qu'Augustin échange avec Jérôme, déjà en exil à Bethléem, à propos du retour au texte hébreu que ce dernier entreprend dans le cadre de l'élaboration de la Vulgate, dévoilent une autre partie des nouveaux interdits : ils frappent non seulement une traduction trop libre, mais encore celle qui pêche par un zèle de fidélité en allant chercher la lettre originale avant sa déformation par la tradition, et qui bouscule l'ordre établi. Dès la Lettre 56, Augustin fait part à Jérôme de son mécontentement de le voir traduire les Saintes Ecritures d'après le texte hébreu ${ }^{33}$. L'entreprise hiéronymienne de traduction met en cause l'autorité de la traduction des Septante, certes altérée par les Vieilles latines, mais elle-même entachée de suspicion d'écarts avec l'original. Or, une retraduction destinée à court-circuiter une traduction précédente faisant autorité est dangereuse : en ne se référant pas à la traduction des Septante, Jérôme, malgré toutes ses précautions, pointe pour le public par sa retraduction le caractère erroné sur certains points de celle des Septante. Cette dernière ayant le statut de version autorisée, c'est donc l'Église instituée qui perd de son autorité lorsqu'on conteste à ce texte sa prééminence. En voulant donner par le biais de son entreprise de traduction une nouvelle interprétation des Saintes Écritures, Jérôme, d'une certaine manière, risque de remettre en cause leur statut.

\section{Conclusion}

En tant qu'elles constituent un ensemble de notions préliminaires à la pensée médiévale de la traduction en Occident, élaborées de surcroît dans une période de changements décisifs d'équilibres, de paradigmes et de valeurs, les réflexions de Jérôme, Augustin et Boèce peuvent être à bon droit qualifiées de prolégomènes aux théories médiévales de la traduction.

La traduction est affaire de descriptions, de prescriptions et de prospections, qui se rapprochent un peu d'observations, de lois et d'expériences. Le traducteur est savant. Mais, du fait des changements d'attitude et des relations avec les institutions, et en premier lieu l'institution ecclésiastique, il se retrouve comme dépossédé de son savoir, qui n'est plus la condition suffisante pour légitimer sa pratique. Le sauveur de textes sous les traits duquel apparaît le traducteur au tournant de l'Antiquité et du Moyen-Âge n'a pas le visage marqué par la superbe : la conquête du passé et la refondation culturelle auxquelles il contribue sont le fruit de l'humilité et de la soumission qu'il a acquise dans ses rapports parfois conflictuels avec l'autorité qui avalise sa tâche. Du fait de Jérôme, Augustin et Boèce, il devient l'expert invisible, à la fois fidèle et infidèle, prolixe et muet, qu'il est encore un peu aujourd'hui.

\footnotetext{
${ }^{33}$ Pour plus de détails, voir Anna SvEnBRO, « Jérôme et Augustin, les prémisses d'une éthique », in Christine Pagnoulle (éd.), Sur le fil : traducteurs et éthique, éthiques du traducteur / Between the Devil and the Deep Blue Sea: Translators and Ethics, Ethics in Translation, Liège : L3, 2010.
} 